International Journal of Modern Physics A

(C) World Scientific Publishing Company

\title{
VECTOR AND AXIAL-VECTOR CORRELATORS IN A INSTANTON-LIKE QUARK MODEL
}

\author{
A.E. Dorokhov \\ Bogoliubov Laboratory of Theoretical Physics, Joint Institute for Nuclear Research, \\ 141980, Dubna, Moscow Region, Russia
}

Received 16 July 2004

\begin{abstract}
The behavior of the vector Adler function at spacelike momenta is studied in the framework of a covariant chiral quark model with instanton-like quark-quark interaction. This function describes the transition between the high energy asymptotically free region of almost massless current quarks to the low energy hadronized regime with massive constituent quarks. The model reproduces the Adler function and $V-A$ correlator extracted from the ALEPH and OPAL data on hadronic $\tau$ lepton decays, transformed into the Euclidean domain via dispersion relations. The leading order contribution from hadronic part of the photon vacuum polarization to the anomalous magnetic moment of the muon, $a_{\mu}^{\text {hvp(1) }}$, is estimated.
\end{abstract}

Keywords: correlators; muon anomalous magnetic moment; topological susceptibility.

In the chiral limit, where the masses of $u, d, s$ light quarks are set to zero, the vector $(V)$ and axial-vector $(A)$ current-current correlation functions in the momentum space (with $-q^{2} \equiv Q^{2} \geq 0$ ) are defined as

$$
\begin{gathered}
\Pi_{\mu \nu}^{J, a b}(q)=i \int d^{4} x e^{i q x} \Pi_{\mu \nu}^{J, a b}(x)=\left(q_{\mu} q_{\nu}-g_{\mu \nu} q^{2}\right) \Pi_{J}\left(Q^{2}\right) \delta^{a b}, \\
\Pi_{\mu \nu}^{J, a b}(x) \quad=\left\langle 0\left|T\left\{J_{\mu}^{a}(x) J_{\nu}^{b}(0)^{\dagger}\right\}\right| 0\right\rangle,
\end{gathered}
$$

where the QCD $V$ and $A$ currents are $J_{\mu}^{a}=\bar{q} \gamma_{\mu} \frac{\lambda^{a}}{\sqrt{2}} q, J_{\mu}^{5 a}=\bar{q} \gamma_{\mu} \gamma_{5} \frac{\lambda^{a}}{\sqrt{2}} q$, and $\lambda^{a}$ are Gell-Mann matrices $\left(\operatorname{tr} \lambda^{a} \lambda^{b}=2 \delta^{a b}\right)$. The momentum-space two-point correlation functions obey (suitably subtracted) dispersion relations,

$$
\Pi_{J}\left(Q^{2}\right)=\int_{0}^{\infty} \frac{d s}{s+Q^{2}} \frac{1}{\pi} \operatorname{Im} \Pi_{J}(s)
$$

where the imaginary parts of the correlators determine the spectral functions $\rho_{J}(s)=4 \pi \Im \Pi_{J}(s+i 0)$. Instead of the correlation function it is more convenient to work with the Adler function defined as

$$
D_{J}\left(Q^{2}\right)=-Q^{2} \frac{d \Pi_{J}\left(Q^{2}\right)}{d Q^{2}}=\frac{1}{4 \pi^{2}} \int_{0}^{\infty} d t \frac{Q^{2}}{\left(t+Q^{2}\right)^{2}} \rho_{J}(t) .
$$

Our goal is to obtain the vector current-current correlator and corresponding Adler function in the low and intermediate regions of momenta by using the effective 
model and then to estimate the leading order hadron vacuum polarization correction to $a_{\mu}$. In $\mathrm{N} \chi \mathrm{QM}$ in the chiral limit the correlators have transverse character

$$
\Pi_{\mu \nu}^{J}\left(Q^{2}\right)=\left(g_{\mu \nu}-\frac{q^{\mu} q^{\nu}}{q^{2}}\right) \Pi_{J}^{\mathrm{N} \chi \mathrm{QM}}\left(Q^{2}\right),
$$

where the polarization functions are given by the sum of the dynamical quark loop, the intermediate (axial-)vector mesons and the higher order mesonic loops contributions

$$
\Pi_{J}^{\mathrm{N} \chi \mathrm{QM}}\left(Q^{2}\right)=\Pi_{J}^{Q \text { Loop }}\left(Q^{2}\right)+\Pi_{J}^{\text {mesons }}\left(Q^{2}\right)+\Pi_{J}^{\chi \text { Loop }}\left(Q^{2}\right),
$$

where the mesons are generated via resummation of quark loop chains.

The dominant contribution to the vector current correlator is given by the dynamical quark loop which was found in 23 with the result

$$
\begin{gathered}
\Pi_{V}^{Q \text { Loop }}\left(Q^{2}\right) \quad=\frac{4 N_{c}}{Q^{2}} \int \frac{d^{4} k}{(2 \pi)^{4}} \frac{1}{D_{+} D_{-}}\left\{M_{+} M_{-}+\left[k_{+} k_{-}-\frac{2}{3} k_{\perp}^{2}\right]_{r e n}\right. \\
\left.+\frac{4}{3} k_{\perp}^{2}\left[\left(M^{(1)}\left(k_{+}, k_{-}\right)\right)^{2}\left(k_{+} k_{-}-M_{+} M_{-}\right)-\left(M^{2}\left(k_{+}, k_{-}\right)\right)^{(1)}\right]\right\}+ \\
+\frac{8 N_{c}}{Q^{2}} \int \frac{d^{4} k}{(2 \pi)^{4}} \frac{M(k)}{D(k)}\left[M^{\prime}(k)-\frac{4}{3} k_{\perp}^{2} M^{(2)}(k, k+Q, k)\right]
\end{gathered}
$$

where the notations $k_{ \pm}=k \pm Q / 2, k_{\perp}^{2}=k_{+} k_{-}-\frac{\left(k_{+} q\right)\left(k_{-} q\right)}{q^{2}}, D(k)=k^{2}+M^{2}(k)$, and $M_{ \pm}=M\left(k_{ \pm}\right), D_{ \pm}=D\left(k_{ \pm}\right)$are used. We also introduce the finite-difference derivatives defined for an arbitrary function $F(k)$ as

$$
F^{(1)}\left(k, k^{\prime}\right)=\frac{F\left(k^{\prime}\right)-F(k)}{k^{2}-k^{2}}, \quad F^{(2)}\left(k, k^{\prime}, k^{\prime \prime}\right)=\frac{F^{(1)}\left(k, k^{\prime \prime}\right)-F^{(1)}\left(k, k^{\prime}\right)}{k^{\prime 2}-k^{2}} .
$$

The difference of the $\mathrm{V}$ and $\mathrm{A}$ correlators is free from any perturbative corrections for massless quarks and very sensitive to the spontaneous breaking of chiral symmetry. The model calculations of the chirality flip combination provides

$$
\begin{gathered}
\Pi_{V-A}^{\mathrm{Loop}}\left(Q^{2}\right)=-\frac{4 N_{c}}{Q^{2}} \int \frac{d^{4} k}{(2 \pi)^{4}} \frac{1}{D_{+} D_{-}}\left\{M_{+} M_{-}+\frac{4}{3} k_{\perp}^{2}\left[-\sqrt{M_{+} M_{-}} M^{(1)}\left(k_{+}, k_{-}\right)+(8)\right.\right. \\
\left.\left.+\left(\sqrt{M^{(1)}}\left(k_{+}, k_{-}\right)\right)^{2}\left(\sqrt{M_{+}} k_{+}+\sqrt{M_{-}} k_{-}\right)^{2}\right]\right\} .
\end{gathered}
$$

and the results extracted from ALEPH experiment 1 is shown in Fig. 1 Oppositely each correlator separately is dominated by perturbative massless quark loop diagram in the high momenta region. In the model calculations this result is reproduced because the dynamical quark mass, $M(k)$ at large virtualities $k^{2}$ vanishes in the chiral limit. The results of the model calculation of the vector Adler function and its experimental conterpart from ALEPH and perturbative QCD asymptotics (see details in 3 ) are presented in Fig. 2

Determination of the Adler function allows us to estimate the leading order hadronic vacuum contribution to the muon anomalous magnetic moment by using the integral representation

$$
a_{\mu}^{\mathrm{hvp}(1)}=\frac{4}{3} \alpha^{2} \int_{0}^{1} d x \frac{(1-x)(2-x)}{x} D_{V}\left(\frac{x^{2}}{1-x} m_{\mu}^{2}\right),
$$




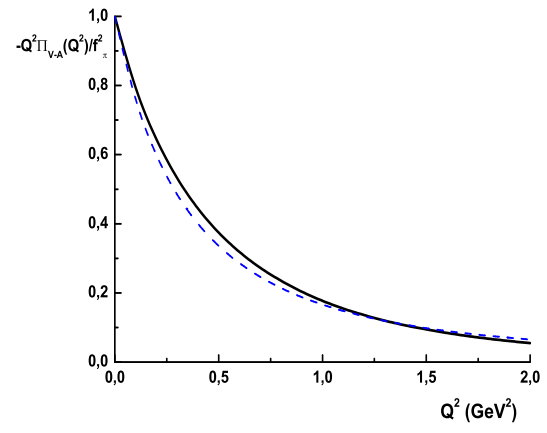

Fig. 1. Normalized $V-A$ correlation function constructed in the $\mathrm{N} \chi \mathrm{QM}$ (solid line) and reconstructed from the ALEPH experimental spectral function (dashed line).

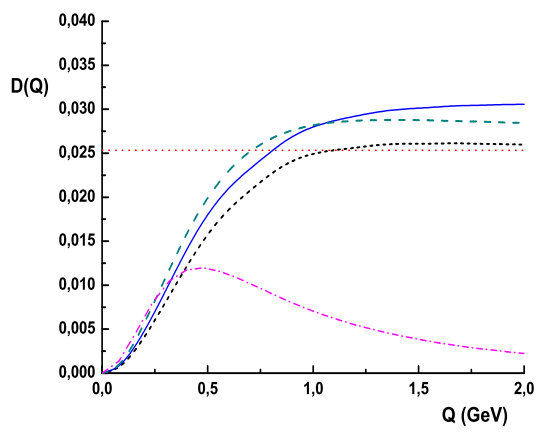

Fig. 2. The Adler function from the $\mathrm{N} \chi \mathrm{QM}$ contributions: dynamical quark loop (short dashed), quark + chiral loops + vector mesons (full line) versus the ALEPH data (dashed). The dash-dotted line is the prediction of the constituent quark model (ENJL) and the dotted line is the asymptotic freedom prediction, $1 / 4 \pi^{2}$.

where the charge factor $\sum Q_{i}^{2}=2 / 3, i=u, d, s$, is taken into account. One gets the model estimate

$$
a_{\mu}^{\mathrm{hvp}(1)}=6.53 \cdot 10^{-8}
$$

which is in a reasonable agreement with the precise phenomenological numbers, found from precise determination of the low energy tail of the total $e^{+} e^{-} \rightarrow$ hadrons and $\tau$ lepton decays cross-sections 4

$$
a_{\mu}^{\mathrm{hvp}(1)}=\left\{\begin{array}{cc}
(6.935 \pm 0.09) \cdot 10^{-8} & e^{+} e^{-}, \\
(7.018 \pm 0.09) \cdot 10^{-8} & \tau .
\end{array}\right.
$$

As by product we estimate also the anomalous magnetic moment of the $\tau$ lepton as

$$
a_{\tau}^{\mathrm{hvp}(1)}=3.06 \cdot 10^{-6} .
$$

We plan to apply the nonlocal chiral quark model to get reliable estimates of the higher order hadronic vacuum polarization to the anomalous magnetic moments of leptons, where there are no precise phenomenological determinations of these contributions.

Ther author thanks for partial support from RFBR Grant no. 02-02-16194.

\section{References}

1. R. Barate et.al. [ALEPH Collaboration], Eur. Phys. J. C 4 (1998) 409.

2. A. E. Dorokhov and W. Broniowski, Eur. Phys. J. C32, 79 (2003).

3. A. E. Dorokhov, arXiv:hep-ph/0405153

4. See for example J.F. de Troconiz, F.J. Yndurain, arXiv:hep-ph/0402285 